Sādhanā, Vol. 26, Part 4, August 2001, pp. 363-370. C Printed in India

\title{
Surface waves in fibre-reinforced anisotropic elastic media
}

\author{
P R SENGUPTA ${ }^{1}$ and SISIR NATH ${ }^{2}$ \\ ${ }^{1}$ 201, Manicktala Main Road, Suite No. 42, Calcutta, 700 054, India \\ ${ }^{2}$ University of Kalyani, Department of Mathematics, Kalyani 741 235, India
}

MS received 6 January 2000; revised 15 January 2001

\begin{abstract}
The aim of this paper is to investigate surface waves in anisotropic fibre-reinforced solid elastic media. First, the theory of general surface waves has been derived and applied to study the particular cases of surface waves Rayleigh, Love and Stoneley types. The wave velocity equations are found to be in agreement with the corresponding classical result when the anisotropic elastic parameters tends to zero. It is important to note that the Rayleigh type of wave velocity in the fibre-reinforced elastic medium increases to a considerable amount in comparison with the Rayleigh wave velocity in isotropic materials.
\end{abstract}

Keywords. Fibre-reinforced medium; surface waves; Rayleigh waves; Love waves; Stoneley waves.

\section{Introduction}

Surface waves have been well recognized in the study of earthquake waves, seismology, geophysics and geodynamics. A good amount of literature is to be found in the standard books of Bullen (1965), Ewing et al (1957), Rayleigh (1885), Love (1911), Stoneley (1924) and Jeffreys (1959), regarding surface waves in classical elasticity. Sengupta and his research collaborators have also studied surface waves (Acharya \& Sengupta 1978; Pal \& Sengupta 1987; Mukherjee \& Sengupta 1991; Das \& Sengupta 1992; Das et al 1994).

In most previous investigations, the effect of reinforcement has been neglected. The idea of continuous self-reinforcement at every point of an elastic solid was introduced by Belfield et al (1983). The characteristic property of a reinforced concrete member is that its components, namely concrete and steel, act together as a single anisotropic unit as long as they remain in the elastic condition, i.e. the two components are bound together so that there can be no relative displacement between them.

In this paper the authors study the propagation of surface waves in fibre-reinforced anisotropic elastic solid media leading to particular cases such as Rayleigh waves, Love waves and Stoneley waves along with numerical results. The results reduce to corresponding classical results when the reinforced elastic parameters tend to zero and the medium becomes isotropic. 


\section{Formulation of the problem}

The constitutive equations for a fibre-reinforced linearly elastic anisotropic medium with respect to a preferred direction $\bar{a}$ are (Belfield et al 1983)

$$
\begin{gathered}
\tau_{i j}=\lambda e_{k k} \delta_{i j}+2 \mu_{T} e_{i j}+\alpha\left(a_{k} a_{m} e_{k m} \delta_{i j}+e_{k k} a_{i} a_{j}\right)+2\left(\mu_{L}-\mu_{T}\right)\left(a_{i} a_{k} e_{k j}+a_{j} a_{k} e_{k i}\right) \\
+\beta\left(a_{k} a_{m} e_{k m} a_{i} a_{j}\right),
\end{gathered}
$$

where $\tau_{i j}$ are components of stress; $e_{i j}=\frac{1}{2}\left(u_{i, j}+u_{j, i}\right)$ are components of strain; $\lambda, \mu_{T}$ are elastic parameters; $\alpha, \beta,\left(\mu_{L}-\mu_{T}\right)$ are reinforced anisotropic elastic parameters; $u_{i}$ are the displacement vectors components and $\bar{a}=\left(a_{1}, a_{2}, a_{3}\right)$, where $a_{1}^{2}+a_{2}^{2}+a_{3}^{2}=1$. If $\bar{a}$ has components that are $(1,0,0)$ so that the preferred direction is the $x_{1}$ axis, (1) simplifies, as given below

$$
\begin{aligned}
& \tau_{11}=\left(\lambda+2 \alpha+4 \mu_{L}-2 \mu_{T}+\beta\right) e_{11}+(\lambda+\alpha) e_{22}+(\lambda+\alpha) e_{33}, \\
& \tau_{22}=(\lambda+\alpha) e_{11}+\left(\lambda+2 \mu_{T}\right) e_{22}+\lambda e_{33}, \\
& \tau_{33}=(\lambda+\alpha) e_{11}+\lambda e_{22}+\left(\lambda+2 \mu_{T}\right) e_{33}, \\
& \tau_{23}=2 \mu_{T} e_{23}, \\
& \tau_{13}=2 \mu_{T} e_{13}, \\
& \tau_{12}=2 \mu_{T} e_{12} .
\end{aligned}
$$

The equations of motion in absence of body forces are

$$
\begin{aligned}
& \frac{\partial \tau_{11}}{\partial x_{1}}+\frac{\partial \tau_{12}}{\partial x_{2}}+\frac{\partial \tau_{13}}{\partial x_{3}}=\rho \frac{\partial^{2} u_{1}}{\partial t^{2}}, \\
& \frac{\partial \tau_{21}}{\partial x_{1}}+\frac{\partial \tau_{22}}{\partial x_{2}}+\frac{\partial \tau_{23}}{\partial x_{3}}=\rho \frac{\partial^{2} u_{2}}{\partial t^{2}}, \\
& \frac{\partial \tau_{31}}{\partial x_{1}}+\frac{\partial \tau_{32}}{\partial x_{2}}+\frac{\partial \tau_{33}}{\partial x_{3}}=\rho \frac{\partial^{2} u_{3}}{\partial t^{2}},
\end{aligned}
$$

where $\rho$ is the density of the elastic medium. Using (2)-(5) and assuming all derivatives with respect to $x_{3}$ vanish, the equations of motion become

$$
\begin{gathered}
\left(\lambda+2 \alpha+4 \mu_{L}-2 \mu_{T}+\beta\right) \frac{\partial^{2} u_{1}}{\partial x_{1}^{2}}+\left(\alpha+\lambda+\mu_{L}\right) \frac{\partial^{2} u_{2}}{\partial x_{1} \partial x_{2}}+\mu_{L} \frac{\partial^{2} u_{1}}{\partial x_{2}^{2}}=\rho \frac{\partial^{2} u_{1}}{\partial t^{2}}, \\
\mu_{L} \frac{\partial^{2} u_{2}}{\partial x_{1}^{2}}+\left(\alpha+\lambda+\mu_{L}\right) \frac{\partial^{2} u_{1}}{\partial x_{1} \partial x_{2}}+\left(\lambda+2 \mu_{T}\right) \frac{\partial^{2} u_{2}}{\partial x_{2}^{2}}=\rho \frac{\partial^{2} u_{3}}{\partial t^{2}} \\
\left(\mu_{L}-\mu_{T}\right) \frac{\partial^{2} u_{3}}{\partial x_{1}^{2}}+\mu_{T}\left(\frac{\partial^{2}}{\partial x_{1}^{2}}+\frac{\partial^{2}}{\partial x_{2}^{2}}\right) u_{3}=\rho \frac{\partial^{2} u_{3}}{\partial t^{2}} .
\end{gathered}
$$

To examine dilatational and rotational disturbances, we introduce two displacement potentials $\phi$ and $\psi$ by the relations

$$
u_{1}=\frac{\partial \phi}{\partial x_{1}}-\frac{\partial \psi}{\partial x_{2}}, \quad u_{2}=\frac{\partial \phi}{\partial x_{2}}-\frac{\partial \psi}{\partial x_{1}} .
$$

The component $u_{3}$ is associated with purely distortional movement. We note that $\phi, \psi$ and $u_{3}$ are respectively associated with $\mathrm{P}$ waves, $\mathrm{SV}$ waves and $\mathrm{SH}$ waves. The symbols have their usual significances. 


\section{General theory and boundary conditions}

The propagation of general surface waves is examined here for a fibre-reinforced elastic solid semi-infinite medium $M$ covered by another fibre-reinforced elastic medium $M_{1}$ $\left(\mathrm{M}_{1}\right.$ above $\mathrm{M}$ and mechanical properties different from $\mathrm{M}$ and which is welded in contact with $\mathrm{M}$ to prevent any relative motion or sliding during the disturbance). We consider an orthogonal Cartesian co-ordinate system $o x_{1} x_{2} x_{3}$ with the origin $o$ at the common plane boundary surface and $o x_{2}$ directed normally into $\mathrm{M}$.

We consider the possibility of a wave travelling in the direction $o x_{1}$ in such a manner that (a) the disturbance is largely confined to the neigbourhood of the boundary and (b) at any instant all particles in any line parallel to $o x_{3}$ have equal displacements. On account of (a) the wave is a surface wave and on account of (b) all the partial derivatives with respect to $x_{3}$ vanish.

Now using (9) in (6) we obtain the following wave equation in M satisfied by $\phi$ and $\psi$ as

$$
\begin{aligned}
& \left(\lambda+2 \alpha+4 \mu_{L}-2 \mu_{T}+\beta\right) \frac{\partial^{2} \phi}{\partial x_{1}^{2}}+\left(\alpha+\lambda+2 \mu_{L}\right) \frac{\partial^{2} \phi}{\partial x_{2}^{2}}=\rho \frac{\partial^{2} \phi}{\partial t^{2}}, \\
& \left(\alpha+3 \mu_{L}+\beta-2 \mu_{T}\right) \frac{\partial^{2} \psi}{\partial x_{1}^{2}}+\mu_{L} \frac{\partial^{2} \psi}{\partial x_{2}^{2}}=\rho \frac{\partial^{2} \psi}{\partial t^{2}},
\end{aligned}
$$

and similar relations in $\mathrm{M}_{1}$ with $\rho, \lambda, \alpha, \mu_{L}, \beta$ replaced by $\rho_{1}, \lambda_{1}, \alpha_{1}, \mu_{L 1}, \beta_{1}$. The general solutions for $\phi$ and $\psi$ must satisfy (7).

\subsection{Boundary conditions}

The boundary conditions for the titled problem are:

(i) the component of displacement at the boundary surface between the media $\mathrm{M}$ and $\mathrm{M}_{1}$ must be continuous at all times and places,

(ii) The stress components $\tau_{21}, \tau_{22}$ and $\tau_{23}$ must be continuous across the interface of $\mathrm{M}$ and $\mathrm{M}_{1}$ at all times and places,

where $\tau_{21}, \tau_{22}$ and $\tau_{23}$ can be written in terms of $\phi$ and $\psi$ in the medium $\mathrm{M}$ with the help of (2) and (9) as

$$
\begin{aligned}
\tau_{21} & =\mu_{L}\left(2 \frac{\partial^{2} \phi}{\partial x_{1} \partial x_{2}}+\frac{\partial^{2} \psi}{\partial x_{1}^{2}}-\frac{\partial^{2} \psi}{\partial x_{2}^{2}}\right), \\
\tau_{22} & =\lambda \nabla^{2} \phi+\alpha\left(\frac{\partial^{2} \phi}{\partial x_{1}^{2}}-\frac{\partial^{2} \psi}{\partial x_{1} \partial x_{2}}\right)+2 \mu_{T}\left(\frac{\partial^{2} \phi}{\partial x_{2}^{2}}+\frac{\partial^{2} \psi}{\partial x_{1} \partial x_{2}}\right), \\
\tau_{23} & =\mu_{T} \frac{\partial u_{3}}{\partial x_{2}}
\end{aligned}
$$

where $\nabla^{2}$ is the two dimensional Laplacian operator given by

$$
\nabla^{2} \equiv \frac{\partial^{2}}{\partial x_{1}^{2}}+\frac{\partial^{2}}{\partial x_{2}^{2}}
$$

Similar relations in $\mathrm{M}_{1}$ with $\mu_{L}, \lambda, \alpha, \mu_{T}$ are replaced by $\mu_{L 1}, \lambda_{1}, \alpha_{1}, \mu_{T 1}$. 


\section{Solution of the problem}

We seek harmonic solutions for (8), (10) and (11) in the form (Bullen 1965),

$$
\phi, \psi, u_{3}=\left\{\bar{\phi}\left(x_{2}\right), \bar{\psi}\left(x_{2}\right), \bar{u}_{3}\left(x_{2}\right)\right\} \exp \left\{i \omega\left(x_{1}-c t\right)\right\},
$$

in $\mathrm{M}$ and similar relations in $\mathrm{M}_{1}$ with the functions $\phi, \psi, u_{3}$ being replaced by $\phi_{1}, \psi_{1}, u_{3}^{1}$. This leads us to a particular solution corresponding to a group of simple harmonic waves of wavelength $2 \pi / \omega$ travelling forward with speed $c$.

It is convenient to introduce $h, r, s$ where

$$
\begin{aligned}
& h=\left\{\frac{\left(\rho c^{2}-\mu_{L}\right)}{\mu_{T}}\right\}^{1 / 2}, \\
& r=\left\{\frac{\left[\rho c^{2}-\left(\lambda+2 \alpha+4 \mu_{L}-2 \mu_{T}+\beta\right)\right]}{\alpha+\lambda+2 \mu_{L}}\right\}^{1 / 2}, \\
& s=\left\{\frac{\left[\rho c^{2}-\left(\alpha+3 \mu_{L}+\beta-2 \mu_{T}\right)\right]}{\mu_{L}}\right\}^{1 / 2},
\end{aligned}
$$

and similar expressions $h_{1}, r_{1}$ and $s_{1}$ for the medium $\mathrm{M}_{1}$. The positive value of the square root being taken in each case.

Now substituting from (13) into (8), (10) and (11) we obtain for the medium M

$$
\begin{aligned}
& u_{3}=C \exp \left[i \omega\left(-h x_{2}+x_{1}-c t\right)\right], \\
& \phi=A \exp \left[i \omega\left(-r x_{2}+x_{1}-c t\right)\right], \\
& \psi=B \exp \left[i \omega\left(-s x_{2}+x_{1}-c t\right)\right],
\end{aligned}
$$

and for the medium $\mathrm{M}_{1}$

$$
\begin{aligned}
& u_{3}^{1}=C_{1} \exp \left[i \omega\left(h_{1} x_{2}+x_{1}-c t\right],\right. \\
& \phi_{1}=A_{1} \exp \left[i \omega\left(r_{1} x_{2}+x_{1}-c t\right],\right. \\
& \psi_{1}=B_{1} \exp \left[i \omega\left(s_{1} x_{2}+x_{1}-c t\right] .\right.
\end{aligned}
$$

In the above, for the effect to be essentially a surface one, each expression must diminish indefinitely with increasing distance from the boundary. This will be the case if each expression contains an exponential factor in which the exponent is real and negative. Hence $h, r, s$ and similarly $h_{1}, r_{1}, s_{1}$ are taken to be imaginary.

Using (15) and (16) in the boundary conditions (i) and (ii) given in $\S(3.1)$ we obtain,

$$
\begin{aligned}
& A+s B=A_{1}-s_{1} B_{1}, \\
& -A r+B=A_{1} r_{1}+B_{1}, \\
& C=C_{1}, \\
& \mu_{L}\left[2 r A+\left(s^{2}-1\right) B\right]=\mu_{L 1}\left[-2 r_{1} A_{1}+\left(s_{1}^{2}-1\right) B_{1}\right], \\
& {\left[(\lambda+\alpha)+r^{2}\left(\lambda+2 \mu_{T}\right)\right] A-\left(2 \mu_{T}-\alpha\right) s B=\left[\left(\lambda_{1}+\alpha_{1}\right)+r_{1}^{2}\left(\lambda_{1}+2 \mu_{T 1}\right] A_{1}\right.} \\
& +\left(2 \mu_{T 1}-\alpha_{1}\right) s_{1} B_{1},
\end{aligned}
$$




$$
-C h \mu_{T}=C_{1} h_{1} \mu_{T 1} .
$$

It follows from (19) and (22) that both $C$ and $C_{1}$ vanish, thus there is no propagation of displacement $u_{3}$. Finally we get the wave velocity equation in the common boundary of the media $\mathrm{M}$ and $\mathrm{M}_{1}$ by eliminating the constants $A, B, A_{1}$ and $B_{1}$ from the equations (17), (18), (20) and (21) as

$\left|\begin{array}{cccc}1 & s & -1 & s_{1} \\ -r & 1 & -r_{1} & -1 \\ 2 \mu_{L} r & \mu_{L}\left(s^{2}-1\right) & 2 r_{1} \mu_{L 1} & -\left(s_{1}^{2}-1\right) \mu_{L 1} \\ {\left[(\lambda+\alpha)+r^{2}\left(\lambda+2 \mu_{T}\right)\right]} & -\left(2 \mu_{T}-\alpha\right) s & -\left[\left(\lambda_{1}+\alpha_{1}\right)+r_{1}^{2}\left(\lambda_{1}+2 \mu_{T 1}\right]\right. & -\left(2 \mu_{T 1}-\alpha_{1}\right) s_{1}\end{array}\right|=0$.

\section{Particular cases}

\subsection{Rayleigh waves}

The particular case of $\mathrm{M}_{1}$ replaced by vacuum was first examined by Rayleigh (1885). The absence of stress over the free surface enables us to replace the right-hand side of (20) and (21) by zero, giving

$$
\begin{gathered}
2 r A+\left(s^{2}-1\right) B=0, \\
{\left[(\lambda+\alpha)+r^{2}\left(\lambda+2 \mu_{T}\right) A-\left(2 \mu_{T}-\alpha\right) s=0 .\right.}
\end{gathered}
$$

Eliminating $A$ and $B$ from (24) and (25) we obtain the Rayleigh type of waves in the fibre-reinforced elastic medium as

$$
\left(1-s^{2}\right)\left[(\lambda+\alpha)+r^{2}\left(\lambda+2 \mu_{T}\right)\right]=2 r s\left(2 \mu_{T}-\alpha\right),
$$

where $r$ and $s$ have been defined in (14).

Now writing $\mu_{L}=\mu_{L}-\mu_{T}+\mu_{T}$ and making $\alpha, \beta$ and $\left|\alpha_{L}-\mu_{T}\right|$ all tend to zero, (26) reduces to the following form,

$$
\left(2-\frac{\rho c^{2}}{\mu_{T}}\right)^{2}=4\left(1-\frac{\rho c^{2}}{\lambda+2 \mu_{T}}\right)^{1 / 2}\left(1-\frac{\rho c^{2}}{\mu_{T}}\right)^{1 / 2},
$$

which is the Rayleigh surface wave in isotropic materials.

5.1a Numerical calculations for Rayleigh waves: The following values of elastic constants and density are considered (Chattopadhyay 1998).

$$
\begin{array}{ll}
\lambda=5.65 \times 10^{9} \mathrm{Nm}^{-2}, & \mu_{L}=5.66 \times 10^{9} \mathrm{Nm}^{-2}, \\
\mu_{T}=2.46 \times 10^{9} \mathrm{Nm}^{-2}, & \alpha=-1.28 \times 10^{9} \mathrm{Nm}^{-2}, \\
\beta=220.90 \times 10^{9} \mathrm{Nm}^{-2}, & \rho=7800 \mathrm{~kg} \mathrm{~m}^{-3} .
\end{array}
$$

Using (26) and the expressions given in (14) we obtain the following value of the wave velocity $c$ as:

$\rho c^{2} / \mu_{L}($ dimensionless $)=40.817316$; 
$\left(\rho c^{2} / \mu_{L}\right)^{1 / 2}=6.38884$

$c^{2}(\mathrm{~m} / \mathrm{s})=296 \times 10^{5}$;

$c(\mathrm{~km} / \mathrm{s})=5.4406$.

The Rayleigh wave propagates very rapidly in fibre-reinforced elastic media according to this theory.

\subsection{Love waves}

For the existence of Love waves we consider a layered semi-infinite medium, in which $\mathrm{M}_{1}$ is obtained by two horizontal plane surfaces, a finite distance $H$ apart, and the medium $\mathrm{M}$ remains as before.

Now we investigate the displacement $u_{3}$ in the direction of $x_{2}$-axis. For the medium M the solution for the displacement component $u_{3}$ remains the same but for the medium $\mathbf{M}_{1}$ we preserve the full solution, since the displacement component $u_{3}$ no longer diminishes with increasing distance from the boundary surface of two media. Hence,

$$
u_{3}^{1}=C_{1} \exp \left[i \omega\left(h_{1} x_{2}+x_{1}-c t\right)\right]+D_{1} \exp \left[i \omega\left(-h_{1} x_{2}+x_{1}-c t\right)\right],
$$

where $h_{1}$ is now not necessarily imaginary. For $\mathrm{M}$ we still have $h$ imaginary. In the present case the boundary conditions are:

(i) $u_{3}$ and $\tau_{23}$ are continuous at $x_{2}=0$

(ii) $\tau_{23}=0$ at $x_{2}=-H$.

Now using (12), (15) and (28) in the above boundary conditions we obtain

$$
\begin{gathered}
C-C_{1}-D_{1}=0, \\
-\mu_{T} h C-\mu_{T 1} h_{1} C_{1}+\mu_{T 1} h_{1} D_{1}=0, \\
\exp \left[-i \omega H h_{1}\right] C_{1}-\exp \left[i \omega H h_{1}\right] D_{1}=0 .
\end{gathered}
$$

Eliminating $C, C_{1}$ and $D_{1}$ from the above equations we obtain

$$
\mu_{T 1} h_{1} \tan \left(\omega H h_{1}\right)+i \mu_{T} h=0 .
$$

Substituting for $h$ and $h_{1}$ from (14) into (32) gives the equation for the velocity $c$ of Love waves, namely

$$
\mu_{T}\left(\frac{\mu_{L}-\rho c^{2}}{\mu_{T}}\right)^{1 / 2}-\mu_{T 1}\left(\frac{\rho_{1} c^{2}-\mu_{L 1}}{\mu_{T 1}}\right)^{1 / 2} \tan \left[\omega H\left(\frac{\rho_{1} c^{2}-\mu_{L 1}}{\mu_{T 1}}\right)^{1 / 2}\right]=0 .
$$

The requirement that $h$ should be imaginary and hence that by (32) $h_{1}$ real is, by (14), satisfied if

$$
\left(\mu_{L 1} / \rho_{1}\right)^{1 / 2}<c<\left(\mu_{L} / \rho\right)^{1 / 2} .
$$

Equation (33) shows that $c$ is dependent on the particular value of $\omega$ and not a fixed constant so that in the present boundary conditions there is dispersion of the general wave form. We see from (33) that if $\omega$ is small, $c \rightarrow\left(\mu_{L} / \rho\right)^{1 / 2}$, while if $\omega$ is large, $c \rightarrow\left(\mu_{L 1} / \rho_{1}\right)^{1 / 2}$. 
5.2a Numerical calculations for Love waves: The upper limit for the wave velocity $c$ given in the inequality (34) for the existence of propagation of Love waves in different elastic solid medium is given below.

Fibre reinforced medium $\left(\mu_{L} / \rho\right)^{1 / 2}(\mathrm{~km} / \mathrm{s})=0.851$;

Lead $(\mu / \rho)^{1 / 2}(\mathrm{~km} / \mathrm{s})=0.69836$;

Copper $(\mu / \rho)^{1 / 2}(\mathrm{~km} / \mathrm{s})=2.08$;

Iron $(\mu / \rho)^{1 / 2}(\mathrm{~km} / \mathrm{s})=3.10$;

Earth crust $(\mathrm{km} / \mathrm{s})=3.2-3.6$.

\subsection{Stoneley waves}

Stoneley investigated a type of surface waves (Stoneley 1924) which are the generalised form of Rayleigh waves propagating at the common boundary of $\mathbf{M}$ and $\mathbf{M}_{1}$. The Stoneley waves in fibre-reinforced elastic media along the common boundary of $\mathrm{M}$ and $\mathrm{M}_{1}$ are, in fact, the same as the general discussion of surface waves presented at the starting and as such the wave velocity is determined by the root of the frequency equation (23). This equation of course reduces once more to the classical result when the parameters for the fibre-reinforced medium tend to zero.

\section{Discussion}

It is clear from the above investigation that the surface waves in the fibre-reinforced medium are affected by the reinforced parameters. In particular, the condition for the existence of propagation of Love waves, given by (34), depends upon the reinforced parameter $\mu_{L}$ and $\mu_{L 1}$. Also all the results reduce to the classical isotropic results when the anisotropic parameters for the fibre-reinforced medium tend to zero (if necessary writing $\mu_{L}=\mu_{L}-\mu_{T}$ and considering $\left|\mu_{L}-\mu_{T}\right| \rightarrow 0$ ).

From $\$ 5.1 \mathrm{a}$, we conclude that the Rayleigh wave velocity in a fibre-reinforced elastic medium is considerably higher than the Rayleigh wave velocity in isotropic media. In this connection, terrestrial Rayleigh wave speed is about $3 \mathrm{~km} / \mathrm{s}$ (Love 1911, p. 160). From $\$ 5.2 \mathrm{a}$, the value of the upper limit of $c$ given in (34) for the stable propagation of Love waves in the fibre-reinforced medium decreases in comparison with the upper limits in other elastic media except lead.

The authors take this opportunity to express their gratitude to the reviewer for his/her valuable comments and suggestions for improving this paper.

\section{References}

Acharya D P, Sengupta P R 1978 Magneto-thermo-elastic surface waves in initially stressed conducting media. Acta Geophys. Polon. A26: 299-311

Belfield A J, Rogers T G, Spencer A J M 1983 Stress in elastic plates reinforced by fibres lying in concentric circles. J. Mech. Phys. Solids 31: 25

Bullen K E 1965 An introduction to the theory of seismology (London: Cambridge University Press) pp 85-99 
Chattopadhyay A, Venkateswarlu R L K 1998 Stresses produced in a fibre-reinforced half-space due to a moving load. Bull. Cal. Math. Soc. 90: 337-342

Das T K, Sengupta P R 1992 Effect of gravity on visco-elastic surface waves in solids involving time rate of stress and strain of first orders. Sädhana 17 : 315-323

Das S C, Acharya D P, Sengupta P R 1994 Magneto-visco-elastic surface waves in stressed conducting media. Sādhanā 19: 337-346

Ewing W M, Jardetzky W S, Press F 1957 Elastic waves in layered media (New York, Toronto, London: McGraw Hill) pp 348-350

Jeffreys H 1959 The earth 4th edn (Cambridge: University Press)

Love A E H 1911 Some problems of geodynamics (New York: Dover)

Markham MF 1970 Measurement of elastic constants of fibre composites, ultrasonic composites 1 p 145

Mukherjee A, Sengupta P R 1991 Surface waves in higher order thermo-visco-elastic media under the influence of gravity. Proc. Natl. Acad. Sci. (India) A61: 411-423

Pal K C, Sengupta P R 1987 Surface waves in visco-elastic media of general type in the presence of thermal field and gravity. Proc. Indian Natl. Sci. Acad. A53: 353-372

Rayleigh Lord 1885 On wave propagation along the plane surface of an elastic solid. Proc. London. Math. Soc. 17: 4-11

Stoneley R 1924 The elastic waves at the surface of separation of two solids. Proc. R. Soc. London A106: 416-420 